\title{
Manifestation purpurique d'un myxome auriculaire infecté
}

\author{
Takashi Yamamoto MD PhD, Kentaro Yamashita MD PhD, Hiroaki Hagiwara MD PhD
}

Citation : CMAJ 2021 March 8;193:E340. doi : 10.1503/cmaj.201351-f

Voir la version anglaise de l'article ici : www.cmaj.ca/lookup/doi/10.1503/cmaj.201351

$\mathbf{U}$

ne femme de 72 ans ayant des antécédents de longue date de fibrillation auriculaire a consulté en dermatologie pour un purpura bilatéral et un œdème aux 2 jambes durant depuis 2 semaines (figure $1 \mathrm{~A}$ ). II y a 22 ans, elle a reçu un diagnostic de myxome géant de l'oreillette droite, mais a refusé de se faire opérer. Il y a 4 mois, elle a subi une intervention dentaire, et au cours des 2 derniers mois, elle a ressenti une fatigue générale et perdu $6 \mathrm{~kg}$. La patiente prenait de l'apixaban en prévention de l'accident vasculaire cérébral. Nous avons observé sur sa peau des taches rouges et violacées non surélevées, allant de l'abdomen aux chevilles et sur la partie supérieure des 2 bras. À l'auscultation, des souffles systoliques et diastoliques étaient audibles au niveau des deuxième et troisième espaces intercostaux. Les analyses de laboratoires ont révélé une leucocytose neutrophile et une hausse du taux de protéine $\mathrm{C}$ réactive; les hémocultures se sont révélées positives à Streptococcus mitis. Les échocardiographies transœsophagiennes ont montré un volumineux myxome pédonculé calcifié arborant des végétations qui emplissait l'oreillette droite et faisait saillie dans l'orifice de la valve tricuspide durant la diastole (voir annexes 1 et 2, accessibles en anglais au www.cmaj.ca/lookup/doi/10.1503/cmaj.201351/tab -related-content, pour une vidéo et des images additionnelles).

Nous avons diagnostiqué une endocardite infectieuse liée au myxome cardiaque préexistant, et amorcé une antibiothérapie avec de l'ampicilline intraveineuse $(200 \mathrm{mg} / \mathrm{kg} / \mathrm{j}$ en 4 doses) et de la gentamicine ( $3 \mathrm{mg} / \mathrm{kg} / \mathrm{j}$ en 1 dose). Au bout de 3 jours, après confirmation de la sensibilité de l'agent causal à la pénicilline $G$, nous avons cessé la gentamicine. Les épreuves de tomodensitométrie avec agent de contraste ont montré 2 petits infiltrats pulmonaires avec cavitation centrale, indicateurs d'emboles pulmonaires septiques. L'examen histologique de la peau a quant à lui montré une vascularite leucocytoclasique. Après 5 jours d'antibiothérapie, le purpura s'est résorbé. La tumeur et les végétations (figure 1B) ont été réséquées et l'examen anatomopathologique a confirmé un myxome cardiaque dégénératif calcifié, avec une infiltration de neutrophiles et des colonies bactériennes. La patiente a continué l'antibiothérapie pendant 4 semaines après la chirurgie et s'est rétablie sans complications.

Environ $8 \%$ de tous les cas d'endocardite infectieuse s'accompagnent de purpura, et le risque de purpura est proportionnel à la taille des lésions valvulaires ${ }^{1}$. Souvent, l'endocardite subaiguë s'accompagne de symptômes non spécifiques, comme chez cette patiente, avant la manifestation du purpura. Le myxome cardiaque infecté est extrêmement rare. On suggère une résection rapide en raison du risque élevé d'embolie ${ }^{2}$. Les cliniciens devraient envisager l'endocardite infectieuse chez les patients qui développent un purpura. 


\section{Références}

1. Lavie G, Henig $\mathrm{O}$, Weber $\mathrm{G}$, et al. Afebrile endocarditis presenting as purpura and acute renal failure. Am J Med 2015;128:e5-6.

2. Yuan SM. Infected cardiac myxoma: an updated review. Braz J Cardiovasc Surg 2015;30:571-8.

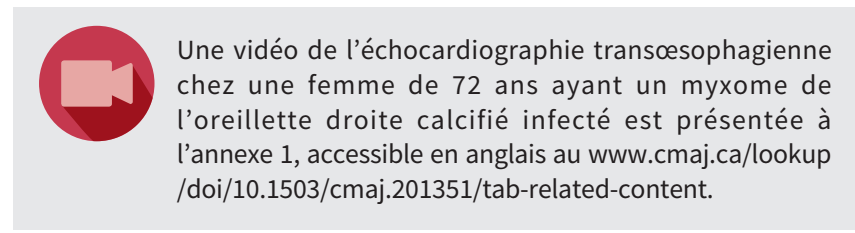

Les images cliniques sont choisies pour leur caractère particulièrement intéressant, classique ou impressionnant. Toute soumission d'image de haute résolution claire et bien identifiée doit être accompagnée d'une légende aux fins de publication. On demande aussi une brève explication (300 mots maximum) de la portée éducative des images, et des références minimales. Le consentement écrit du patient au regard de la publication doit être obtenu avant la soumission.
Intérêts concurrents : Aucun intérêt concurrent n'a été déclaré.

Cet article a été révisé par des pairs.

Les auteurs ont obtenu le consentement de la patiente.

Affiliations : Départements de cardiologie (Yamamoto, Yamashita) et de chirurgie cardiovasculaire (Hagiwara), Organisation nationale des hôpitaux, Centre médical Nagoya, Aichi, Japon.

Propriété intellectuelle du contenu : Il s'agit d'un article en libre accès distribué conformément aux modalités de la licence Creative Commons Attributions (CC BY-NC-ND 4.0), qui permet l'utilisation, la diffusion et la reproduction dans tout médium à la condition que la publication originale soit adéquatement citée, que l'utilisation se fasse à des fins non commerciales (c.-à-d. recherche ou éducation) et qu'aucune modification ni adaptation n'y soit apportée. Voir : https://creativecommons.org/licenses/by-nc-nd/4.0/deed.fr.

Remerciements : Les auteurs remercient le $D^{r}$ Fumito Anno (Département de médecine interne générale) et le $D^{r}$ Shu Ichihara (Département d'anatomopathologie, Organisation nationale des hôpitaux, Centre médical Nagoya) pour leurs fructueuses discussions.

Correspondance : Takashi Yamamoto, takashi-y@hotmail.co.jp 